\title{
MRI EVALUATION OF PERIANAL FISTULAS
}

\author{
Flora Juanita Edwin ${ }^{1}$, Baskar Thangadurai Murugan ${ }^{2}$
}

${ }^{1}$ Associate Professor, Department of Radiodiagnosis, Government Thoothukudi Medical College.

${ }^{2}$ Assistant Professor, Government Thoothukudi Medical College.

\section{ABSTRACT}

\section{BACKGROUND}

Magnetic resonance imaging accurately demonstrates the anatomy of the perianal region fistulas, their relationship with ischiorectal fossa, classifying fistulas into five types. Magnetic resonance imaging depicts infectious foci in the perianal region and helps to reduce postoperative recurrence.

The aim of the study is to evaluate the role of magnetic resonance imaging (MRI) in viewing and classifying perianal fistulas.

\section{MATERIALS AND METHODS}

This study is a descriptive study with 30 patients referred from Surgery Department. MRI was performed and was correlated with operative findings. A GE 1.5 Tesla MRI scanner was used.

\section{RESULTS}

18 cases had intersphincteric fistula, 8 cases had trans-sphincteric type, 2 cases had suprasphincteric and 2 cases had extrasphincteric types. Abscess collections have been found in $15 \%$ patients.

\section{CONCLUSION}

MRI is a reliable diagnostic modality in the classification of perianal fistulas and can be an excellent diagnostic guide for successful surgical interventions with the aim to reduce the number of recurrences. Its advantage is that fistulas and abscesses are visible without the need to apply any contrast medium.

\section{KEYWORDS}

MRI (Magnetic Resonance Imaging), T1-T1 Weighted Imaging, PD-Proton Density, T2-T2 Weighted Imaging.

HOW TO CITE THIS ARTICLE: Edwin FJ, Murugan BT. MRI evaluation of perianal fistulas. J. Evolution Med. Dent. Sci. 2017;6(86): 5951-5954, DOI: $10.14260 /$ jemds/2017/1295

\section{BACKGROUND}

As per definition, a fistula is an abnormal passage connecting two epithelial surfaces. Goodsall describes the fistulous passage in detail, and Parks' classification shows the most practical significance until nowadays. The classification refers to classifying fistulas as intersphincteric, trans-sphincteric, suprasphincteric and extrasphincteric. Magnetic resonance imaging accurately demonstrates the anatomy of the perianal region fistulas, their relationship with ischiorectal fossa, classifying fistulas into five types. Magnetic resonance imaging depicts infectious foci in the perianal region and helps to reduce postoperative recurrence.

The aim of the study is to evaluate the role of magnetic resonance imaging (MRI) in viewing and classifying perianal fistulas.

\section{MATERIALS AND METHODS}

The study is a descriptive study with study population comprising of 30 patients whose MRI studies (done between June 2016 and June 2017) were evaluated. The patients had been referred to the MRI unit for MR fistulography.

'Financial or Other Competing Interest': None.

Submission 27-07-2017, Peer Review 13-10-2017,

Acceptance 19-10-2017, Published 26-10-2017.

Corresponding Author:

Dr. Bhaskar Thangadurai Murgan,

Assistant Professor

Thoothukudi Medical College.

E-mail: durai.baskar923@gmail.com

DOI: $10.14260 /$ jemds/2017/1295
All MRI studies were carried out on a 1.5-T MRI system (GE 1.5T). The sequences evaluated were: Axial T1 TSE; Axial fat-suppressed T2W; Axial post-contrast T1W, Coronal T1W TSE; Coronal T2W fat-suppressed; and coronal post-contrast T1W.

\section{RESULTS}

30 cases were evaluated for the site of the primary tract, presence/absence of external sphincter involvement, and the location of the internal openings.

28 cases had a primary fistula with five had previously undergone perianal abscess drainage.

Out of a total of 30 cases in the study, 18 cases had intersphincteric fistula, 8 cases had trans-sphincteric type, 2 cases had suprasphincteric and 2 cases had extrasphincteric type.

Type 1 simple fistulas were seen in $50 \%$ of the cases.

Horse shoe type of fistulas were seen in $15 \%$ of the cases.

Most of the recurrent cases were suprasphincteric type cases.

Most of the cases had simple fistulas and few had complex types.

Abscess collections have been found in 15\% patients.

\begin{tabular}{|c|c|}
\hline $\begin{array}{c}\text { Types of Perianal } \\
\text { Fistulas }\end{array}$ & $\begin{array}{c}\text { Percentage } \\
\text { (Cases) }\end{array}$ \\
\hline Intersphincteric & $18(60 \%)$ \\
\hline Trans-sphincteric & $8(26.6 \%)$ \\
\hline Suprasphincteric & $2(6.6 \%)$ \\
\hline Extrasphincteric & $2(6.6 \%)$ \\
\hline
\end{tabular}




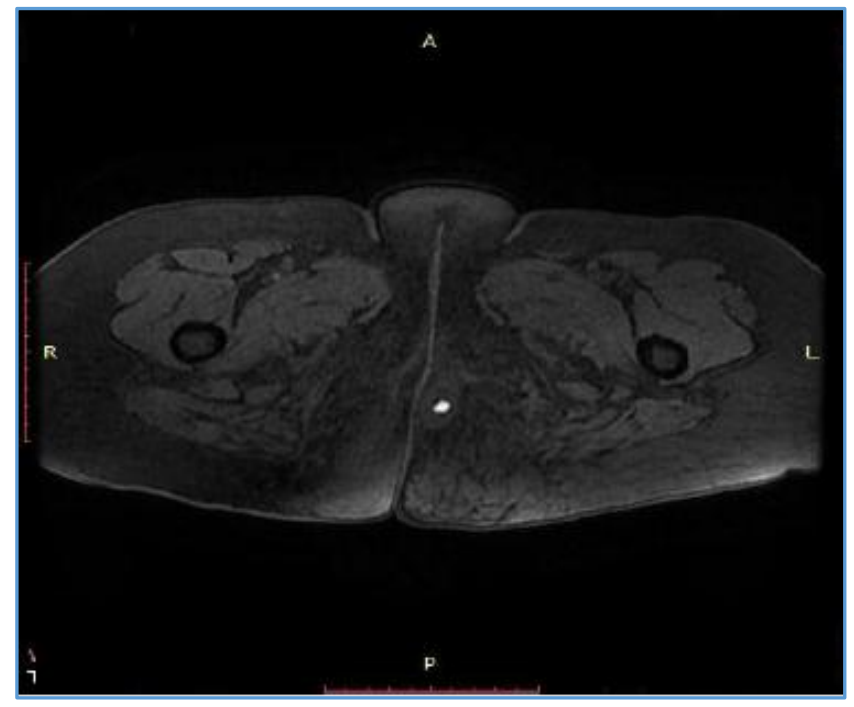

Figure 1. Axial Stir Showing Simple Linear Type 1 Perianal Fistula

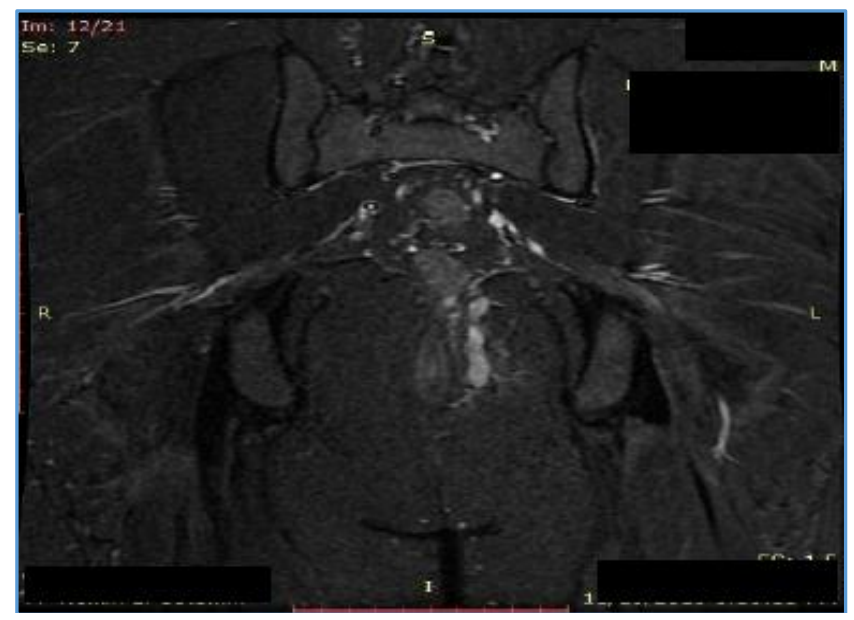

Figure 2. Coronal Stir Showing Suprasphincteric Fistula

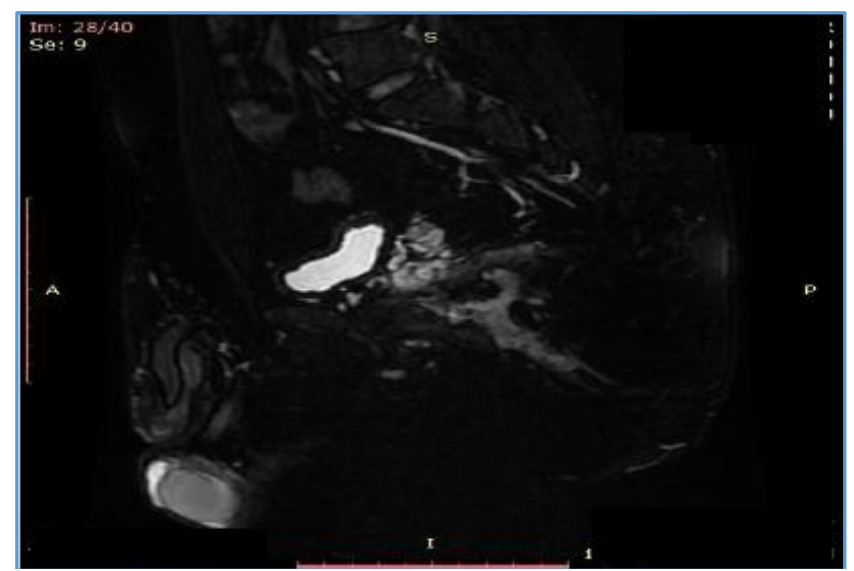

Figure 3. Sagittal Stir Showing Suprasphincteric Fistula

\section{DISCUSSION}

MRI provides excellent soft tissue contrast resolution and the multiplanar display of anatomy.

In one of the early studies on MR fistulography, Lunniss et al reported a concordance rate of $86 \%-88 \%$ between MRI and surgical findings.[1] Subsequent studies have suggested that MRI is more sensitive than even surgical exploration of the tract.[2],[3] MRI is especially useful in patients with fistulas associated with Crohn disease and those with recurrent fistulas, ${ }^{[4]}$ as these entities are associated with branching fistulous tracts. Missed extensions are the commonest cause of recurrence. [5]

T2W images provide good contrast between the tract and the fibrous wall of the fistula, providing good delineation of the layers of the anal sphincter.[6],[7]

With MRI providing excellent anatomical details and relations of fistulous tracts and their associated abscesses, an MR-based classification is now used that categorises perianal fistulas into five grades. Grade 1 fistulas are simple linear intersphincteric fistulas that traverse the intersphincteric plane to the perianal skin, completely confined by the external sphincter. There is no branching within the sphincteric complex or extension into the ischioanal space.

Intersphincteric fistulas with an associated abscess or secondary tract confined by the sphincteric complex are considered Grade 2 fistulas. Secondary tracts ramify in the ipsilateral intersphincteric space or may cross the midline forming horse-shoe tracts. Grade 3 trans-sphincteric fistulas pierce both layers of the sphincter complex and track down to the skin through the ischioanal fossa. Those associated with abscess or secondary tract in the ischioanal fossa are considered as Grade 4. Grade 5 supralevator and translevator disease may have tracts extending cranially in the intersphincteric plane above the levator ani muscle and then piercing downward through the ischioanal fossa referred to as suprasphincteric fistulas or could be extrasphincteric as a result of extensions of pelvic disease.

In case of multiple tracts, images should be assessed for communication among the tracts. Associated secondary extensions or abscesses are defined by their anatomical location: ischioanal, intersphincteric or supralevator.

Horse-shoe tract is formed when they cross the midline to the contralateral side. Few centres including our own have shown improved visualisation of fistulous tracts and their relationship to the surrounding perianal structures using MR fistulogram, a procedure involving injection of diluted gadolinium into the tract using an infant feeding tube. This technique has found usefulness especially in evaluating complex fistulas and tracts with relatively sparse secretion. MRI is also useful in assessment following surgical intervention. In the immediate postoperative period, haemorrhage may appear hyperintense on unenhanced T1weighted images differentiating it from residual tract. Similar hyperintensity may be seen in fat containing 'grafts' used for filling cavities following restorative surgery.

The most appropriate protocol used for evaluation of perianal fistulas consists of the following sequences: oblique axial T1-weighted FSE, oblique axial T2-weighted FSE, and oblique axial and oblique coronal fat-suppressed T1weighted FSE with gadolinium-based contrast material, oriented perpendicular or parallel to the long axis of the anal canal. The planes used are obliquely axial and obliquely coronal relative to the pelvis, but these planes are truly orthogonal and parallel relative to the anal canal and thus suitable for correct evaluation of perianal fistulas. It is not appropriate to use the terms axial and coronal to refer to these planes; their use is not correct in terms of the orientation of the planes relative to the pelvis.

Fat-suppressed T2-weighted sequences such as short inversion time inversion-recovery (STIR) or frequency- 
selective fat-saturated T2-weighted FSE may be used to increase the conspicuity of fluid-containing tracts or abscesses. Frequency-selective fat suppression should be used with the T2-weighted FSE sequence because the high signal intensity of fat can hide active fistulous tracts or abscesses, which also have high signal intensity. On fatsuppressed T2-weighted images, fluid, pus, and granulation tissue are seen as areas of high signal intensity on a background of low-signal-intensity fat.

The STIR sequence provides good suppression of fat signal, but the images tend to be of poorer spatial resolution than frequency-selective fat-saturated T2-weighted FSE images, which provide better visualisation of anatomic details. On frequency-selective fat-saturated T2-weighted FSE images, the efficiency of fat suppression may not be uniform; therefore, care must be taken to place the patient close to the centre of the magnet to maximise the homogeneity of fat suppression. In a prospective study of 42 patients, STIR imaging failed to demonstrate secondary tracts and did not reveal small residual perianal abscesses from perianal inflammation, making it less suitable for demonstration of fluid collections or extensions than are T1-weighted sequences with intravenous contrast material.

Selection of the MR imaging protocol is particularly important in postoperative patients. Local alteration of the magnetic field by a foreign body could induce susceptibility artifact. The term susceptibility refers to the ability of a substance to distort the static magnetic field. The artifact occurs at the interface of substances with differing magnetic susceptibilities. MR imaging artifacts in the postoperative patient may be due to suture artifact, like a seton, placed through the fistula tract. Silk is one of the most commonly used sutures, and the susceptibility artifact induced by silk is the most prominent relative to those from all the other types of sutures.

Refocused pulses, usually $180^{\circ}$, correct many sources of dephasing, making FSE imaging effective for minimising susceptibility artifact. However, if frequency-selective fat saturation techniques are used, variations of the regional magnetic field surrounding the seton may create an inhomogeneous magnetic field, with resultant areas of suboptimal fat saturation.

Susceptibility-induced field inhomogeneity makes frequency-selective fat saturation difficult, and frequencyselective fat saturation exacerbates susceptibility artifact. One should consider not using frequency-selective fat saturation when severe susceptibility artifact is expected.

The STIR sequence is an effective alternative method of suppressing fat signal and is less dependent on the homogeneity of the main magnetic field. STIR relies on T1 relaxation differences instead of precessional frequency differences to cancel the signal from fat, thereby minimising susceptibility effects during fat-suppressed imaging. This sequence is less sensitive to magnetic field in homogeneities and can be used with low-field-strength magnets.

T2-weighted sequences are essential in evaluation of the pelvic region because they provide excellent soft-tissue contrast of the pelvic organs.

The two-dimensional (2D) spin-echo-based T2-weighted sequence, performed in multiple planes, plays a crucial role in basic standardised protocols for pelvic MR imaging.
New sequences will offer opportunities to improve efficiency and diagnostic capability. Three dimensional (3D) T2-weighted turbo spin-echo (TSE) sequences can provide source data for post-processing reformation of images into any desired plane. Therefore, a single 3D T2-weighted sequence with post-processing reformation of images in the axial, coronal, and sagittal planes can potentially replace 2D sequences in those three planes, decreasing the number of sequences performed from three to one.

The 3D imaging technique has several advantages over 2D imaging: There is no operator dependence in acquiring images in any obliquity, a larger volume can be covered, thinner sections without intersection gaps can be obtained, a higher signal-to-noise ratio can be achieved, and imaging time can be reduced. The 3D T2-weighted TSE sequence has been studied for its usefulness in evaluation of rectal cancer, the body trunk, and the female pelvis. However, to our knowledge, use of the 3D T2-weighted TSE sequence for imaging of perianal fistulas has not been reported.

Another new diagnostic tool for detection of perianal fistulas is digital subtraction MR fistulography. Subtraction MR fistulography is based on abnormal enhancement of the inflamed fibrous walls of fistulas or abscesses on T1weighted images after intravenous administration of contrast material. In a prospective study of 36 patients, results of subtraction MR fistulography were compared with surgical findings in patients with anal fistulas or abscesses.

The examination protocol was defined as subtraction MR fistulography because image subtraction resulted in visualisation of fistulas as high-signal-intensity tubular structures containing varying degrees of low-signal-intensity fluid; the surrounding fat appeared dark. The authors demonstrated that high-resolution subtraction MR fistulography, which consisted of a high-resolution 3D T1weighted gradient-echo sequence and the image subtraction technique, was useful in diagnosis of anal fistulas.

Use of diffusion-weighted sequences for evaluation of perianal fistulas has been reported. Diffusion-weighted imaging reflects changes in water mobility caused by interactions with cell membranes, macromolecules, and alterations of the tissue environment. Because inflammatory tissues usually have high signal intensity at diffusionweighted imaging, it may be a promising sequence for diagnosis of anal fistulas, particularly as an adjunct to T2weighted imaging in patients with risk factors having to do with adverse effects of contrast material: previous allergic reactions or impaired renal function with concern about development of nephrogenic systemic fibrosis.

A recent development is use of dynamic contrastenhanced MR imaging for determining the degree of activity in perianal Crohn disease. With this technique, 2D T1weighted sequences are performed and time-signal intensity curves are obtained to determine whether a fistula is active by measuring the volume of enhancing pixels. It was concluded that dynamic contrast-enhanced MR imaging might be helpful in selecting a subpopulation of patients with perianal Crohn disease who should be monitored more closely for development of more extensive disease.

Finally, another development is the possibility of evaluating perianal fistulas by using 3.0-T imaging. In theory, higher-field-strength MR imaging provides a better signal-tonoise ratio, which can be used to achieve increased temporal 
resolution, decreased imaging time, and increased spatial resolution. The increased spatial resolution has the potential to improve lesion visibility. The finer detail of reformatted images can help in characterisation of perianal fistulas.

In 3.0-T imaging, the intrinsic tissue relaxation kinetics defined by $\mathrm{T} 1, \mathrm{~T} 2$, and $\mathrm{T} 2 *$ values vary slightly at higher field strengths, causing a decrease in intrinsic image contrast. Although the intrinsic image contrast is decreased, the T1shortening effect of gadolinium relative to that in adjacent tissues is more pronounced. Therefore, a perianal fistula could be highlighted more markedly from adjacent tissues and the outlines of the fistula tract could be more readily apparent at $3.0 \mathrm{~T}$ than at $1.5 \mathrm{~T}$.

Gadolinium-enhanced T1W images are useful to differentiate a fluid-filled tract from an area of inflammation. ${ }^{[8]}$ Abscesses are well depicted on postgadolinium images.

The exact location of the primary tract (Ischioanal or intersphincteric) is most easily visualised on axial images; the presence of disruption of the external anal sphincter differentiates a trans-sphincteric fistula from an intersphincteric one. The internal opening of the fistula is also best seen in this plane.

Coronal images depict the levator plane, thereby allowing differentiation of supralevator from infralevator infection. A combination of an axial and a longitudinal series (coronal, sagittal, or radial) will provide all the necessary details. [9]

To summarise, evaluation of an enhanced T1W image, in conjunction with a fat-suppressed $\mathrm{T} 2 \mathrm{~W}$ image, provides most of the details necessary for accurate evaluation of perianal fistulas.

\section{CONCLUSION}

By providing high resolution images of the perianal anatomy and by exquisitely depicting perianal sinus tracts with their associated secondary ramifications and abscesses, MRI far surpasses all other imaging modalities in evaluating perianal fistulas. Contrast administration adds further information and may aid differentiation between active and inactive tracts and by outlining tracts not routinely visualised. The ability of MRI to accurately classify perianal fistulas preoperatively and sensitivity in detecting distal foci of infection may aid the planning of and potential success of surgery.

\section{REFERENCES}

[1] Lunniss PJ, Armstrong P, Barker PG, et al. Magnetic resonance imaging of the anal fistulae. Lancet 1992;340(8816):394-6.

[2] Spencer JA, Chapple K, Wilson D, et al. Outcome after surgery for perianal fistula: predictive value of MR imaging. AJR Am J Roentgenol 1998;171(2):403-6.

[3] Beckingham IJ, Spencer JA, Ward J, et al. Prospective evaluation of dynamic contrast enhanced MRI in the evaluation of fistula in ano. $\mathrm{Br} J$ Surg 1996;83(10):1396-8.

[4] Beets-Tan RG, Beets GL, van der Hoop AG, et al. Preoperative MR imaging of anal fistulas: does it really help the surgeon. Radiology 2001;218(1):75-84.

[5] Lilius HG. Fistula-in-ano, an investigation of human foetal anal ducts and intramuscular glands and a clinical study of 150 patients. Acta Chir Scand Suppl 1968;383:7-88.

[6] Stoker J, Rociu E, Zwamborn AW, et al. Endoluminal MR imaging of the rectum and anus: technique, application and pitfalls. Radiographics 1999;19(2):383-98.

[7] Maier AG, Funovics MA, Kreuzer SH, et al. Evaluation of perianal sepsis: comparison of anal endosonography and MRI. J Magn Reson Imaging 2001;14(3):254-60.

[8] Spencer JA, Ward J, Beckingham IJ, et al. Dynamic contrast enhanced MR imaging of perianal fistulas. AJR Am J Roentgenol 1996;167(3):735-41.

[9] Stoker J, Fa VE, Eijkemans MJ, et al. Endoanal MRI of perianal fistulas: the optimal imaging planes. Eur Radiol 1998;8(7):1212-6. 